\title{
Wildfire history of the boreal forest of southwestern Yakutia (Siberia) over the last two millennia documented by a lake-sedimentary charcoal record
}

\section{Supplement}

Overview of the different charcoal size classes (S1-S3) and morphotype classes (S4-S6) in both the classic and robust analysis approach, separated by vertical dashed lines representing the different phases of the fire regime.

For each figure: (a) Classic CHAR peak component (dark grey bars = signal, light grey bars = noise, dashed horizontal line = threshold). (b) SNI of the classic CHAR peak component after Kelly et al. (2011) (red horizontal line = SNI cutoff value of 3). (c) Classic CHAR sum (black line = interpolated CHAR, blue line = LOESS representing the CHAR background component, red vertical lines = fire episodes with $\mathrm{SNI}>3$, grey vertical lines = fire episodes with $\mathrm{SNI}<3$ ). (d) Robust CHAR background component. (e) Robust CHAR peak component (red areas = above-average values).

(f) Robust CHAR sum. For (d)-(f): black line = median, grey area = interquartile range.

\section{S1: Size class $150-300 \mu \mathrm{m}$}

S2: Size class $300-500 \mu \mathrm{m}$

S3: Size class $>500 \mu \mathrm{m}$

S4: Angular morphotypes (S, B, C)

S5: Elongated morphotypes (F, D, E)

S6: Irregular morphotypes (M, P, X) 
S1: Size class $150-300 \mu \mathrm{m}$

Phase 1

Phase 2

Phase 3

Phase 4

(a) Classic CHAR peak

气 0.5

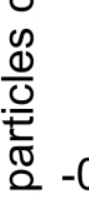

$\propto-0.5$

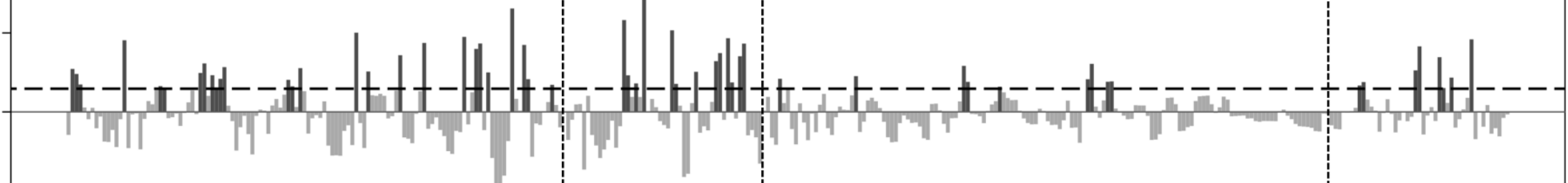

(b) SNI

(c) Classic CHAR

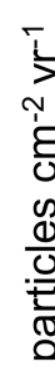

(e) Robust CHAR peak

(d) Robust CHAR background
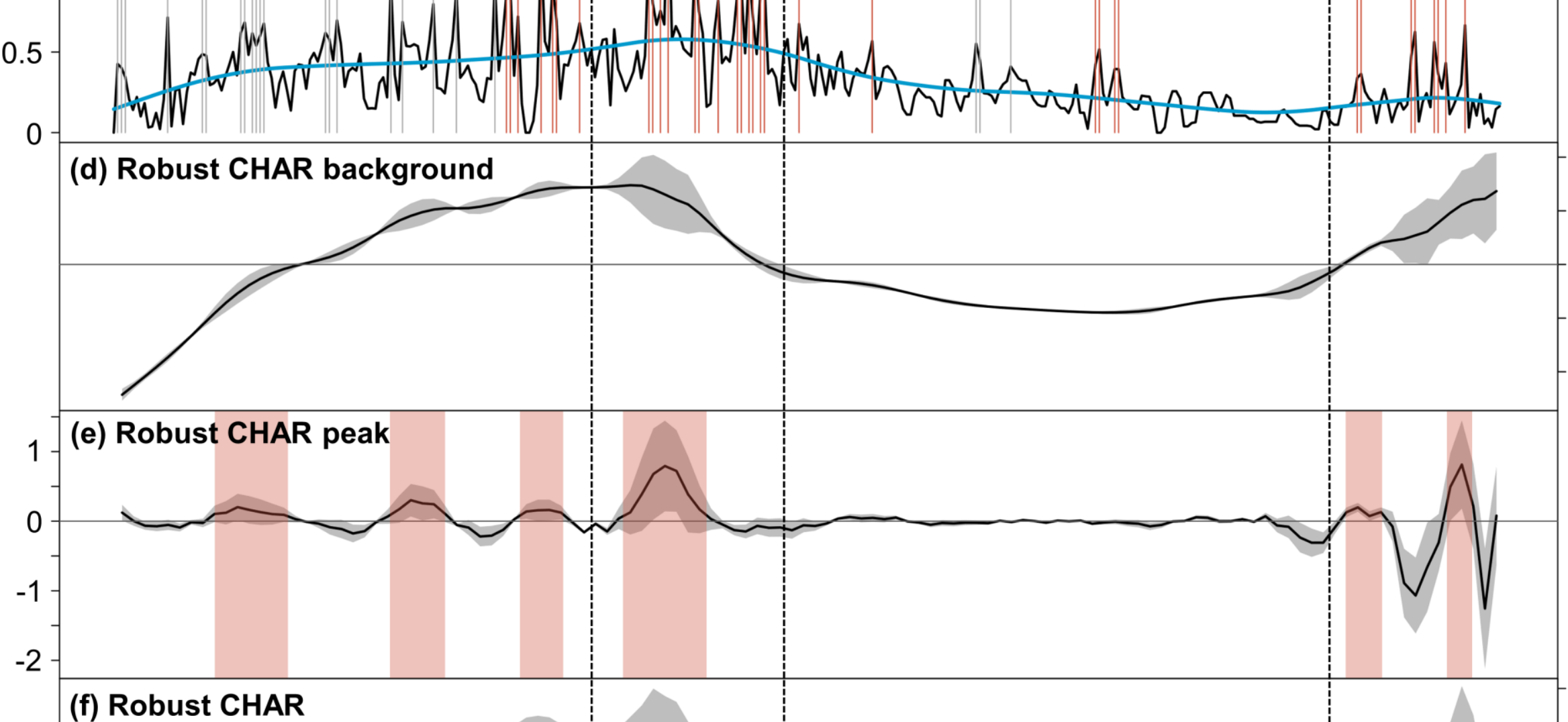

(f) Robust CHAR
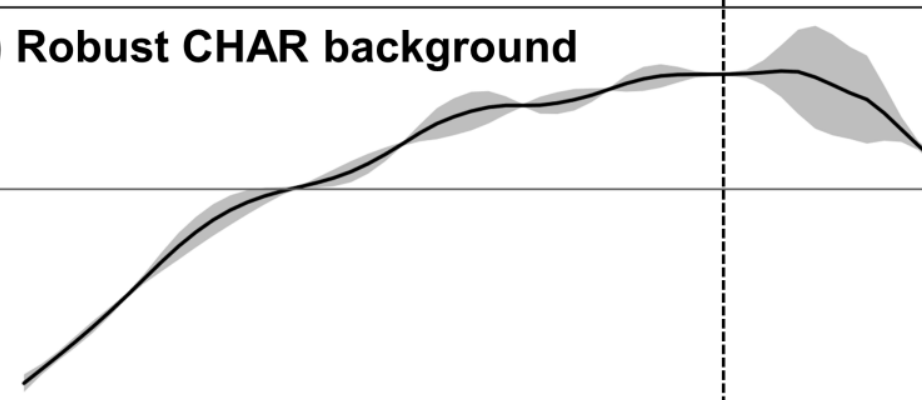

Mñvans

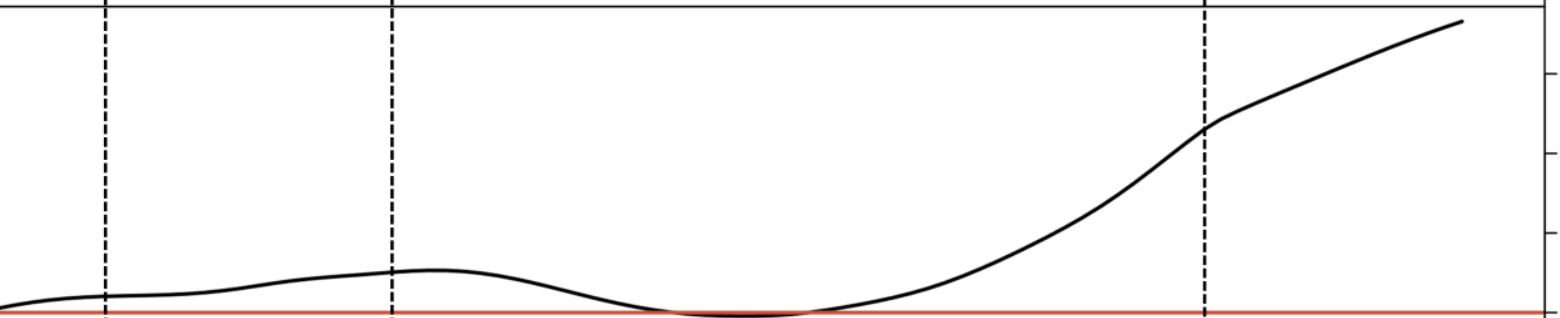


S3: Size class $>500 \mu \mathrm{m}$

Phase 1

Phase 2

Phase 3

Phase 4

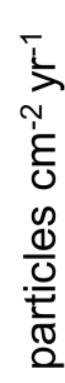

(a) Classic CHAR peak

0.5

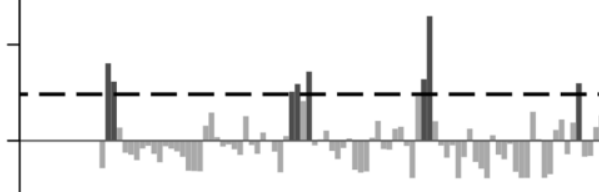

(b) SNI

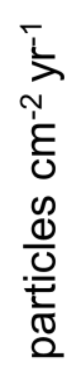

(c) Classic CHAR

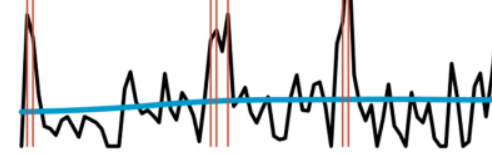

(d) Robust CHAR background

0.5

4

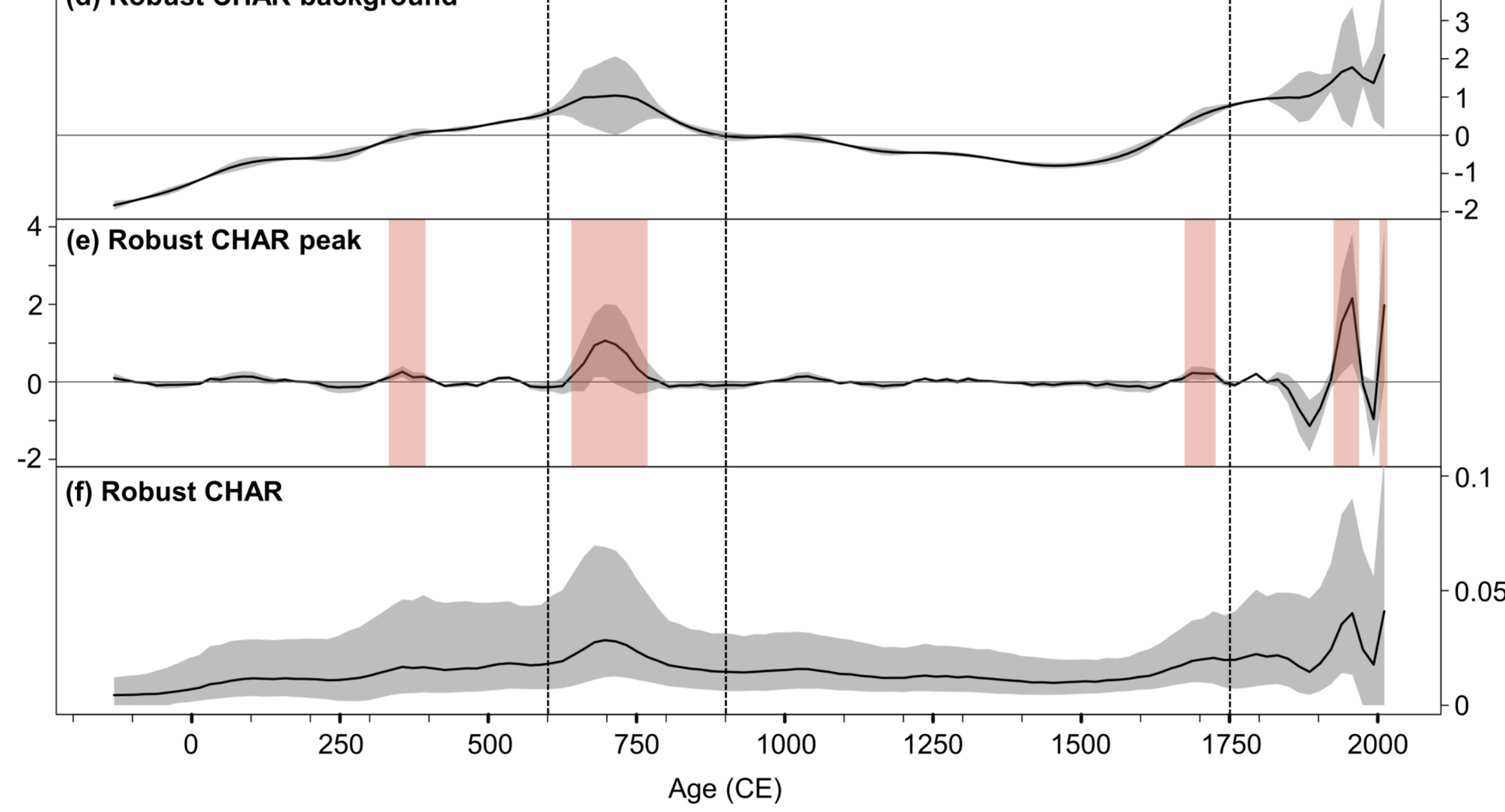

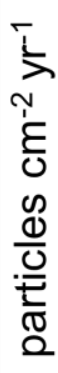


S4: Angular morphotypes

Phase 1

Phase 2

Phase 3

Phase 4

0.6 (a) Classic CHAR peak

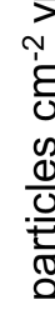

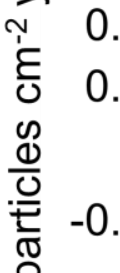

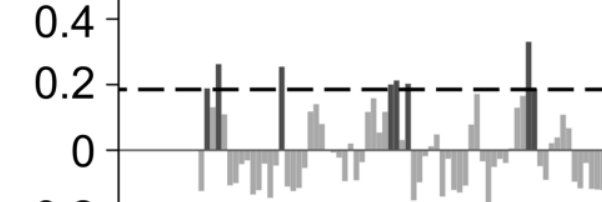

(b) SNI

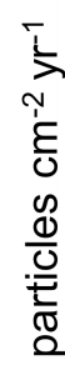

(c) Classic CHAR

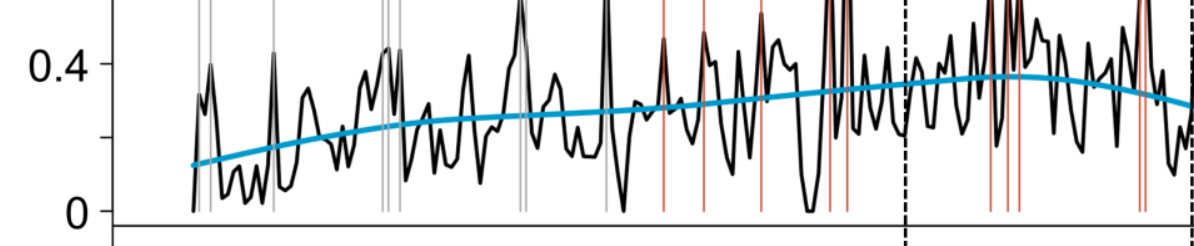

(d) Robust CHAR background

(e) Robust CHAR peak

0
0
0
$N$
$N$

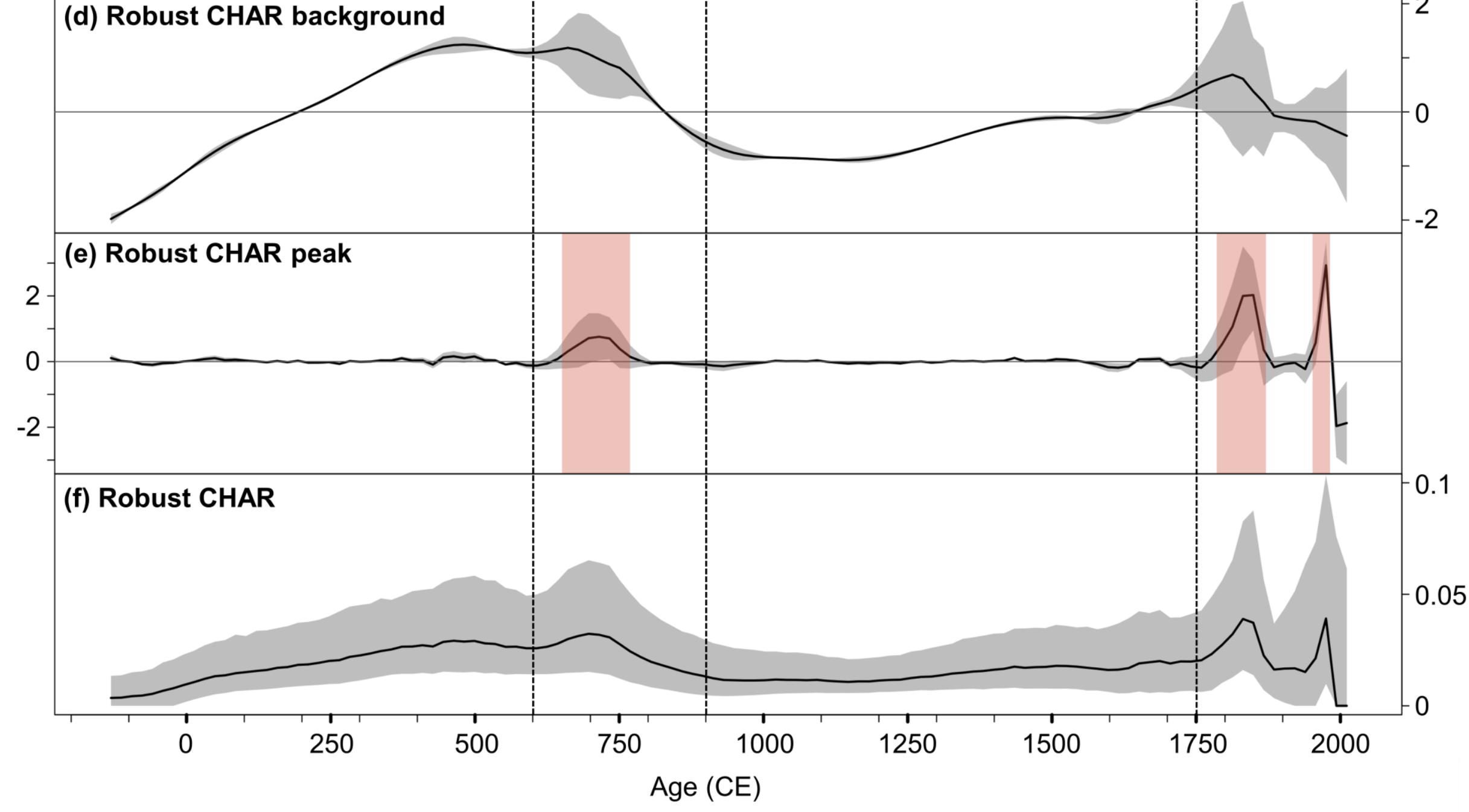

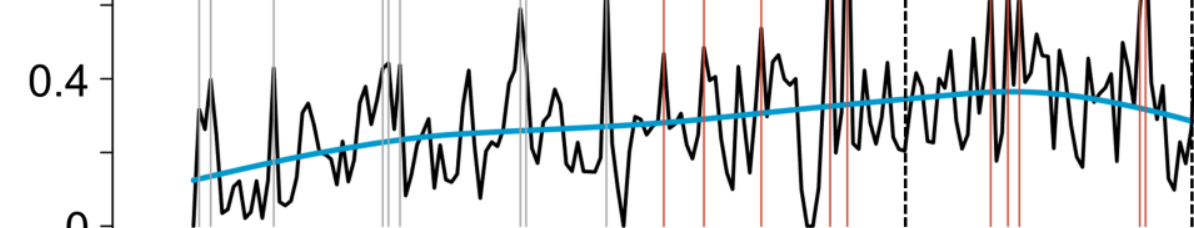
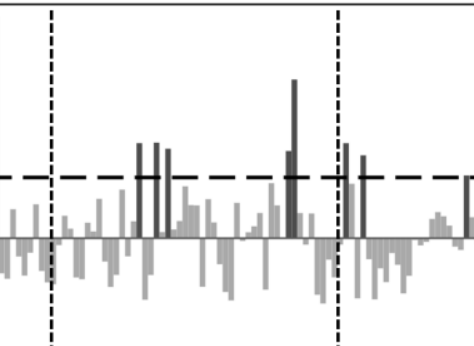

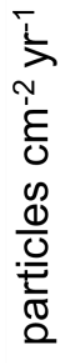

0.1

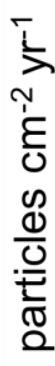


S6: Irregular morphotypes

Phase 1

Phase 2

Phase 3

Phase 4

(a) Classic CHAR peak

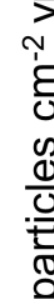

$-0.2$

(b) SNI

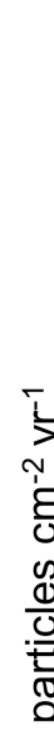

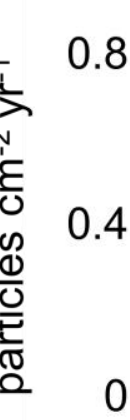

(c) Classic CHAR

(d) Robust CHAR background

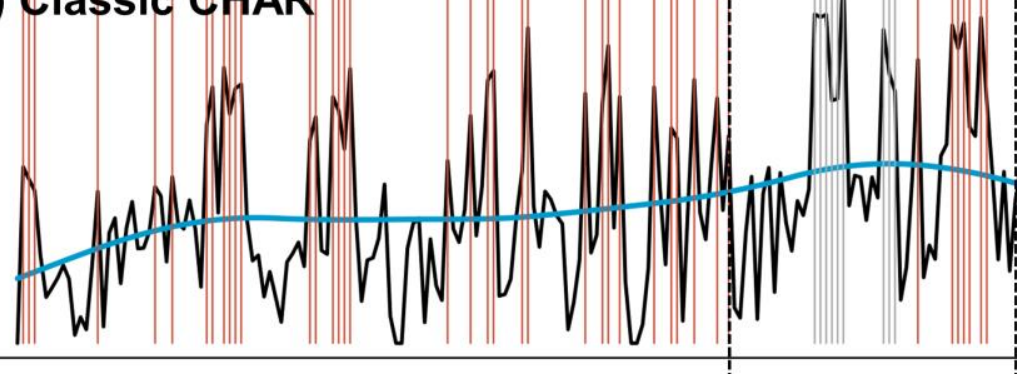

0
0
0
$N$
$N$

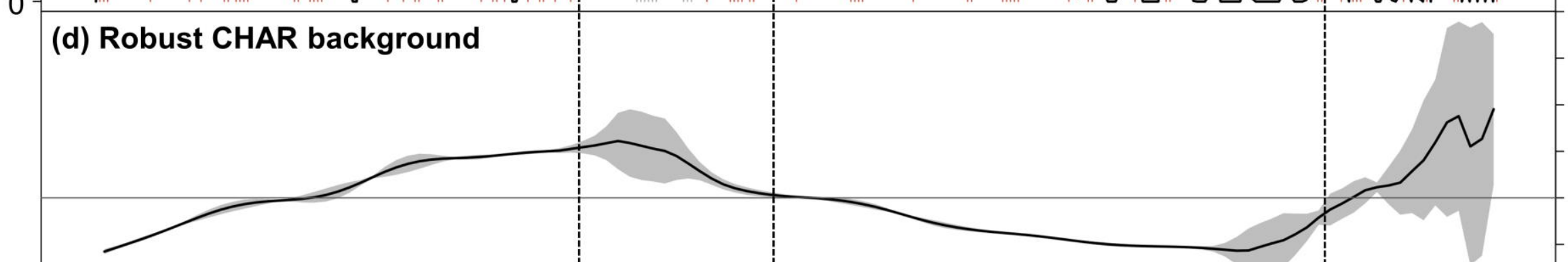

(e) Robust CHAR peak

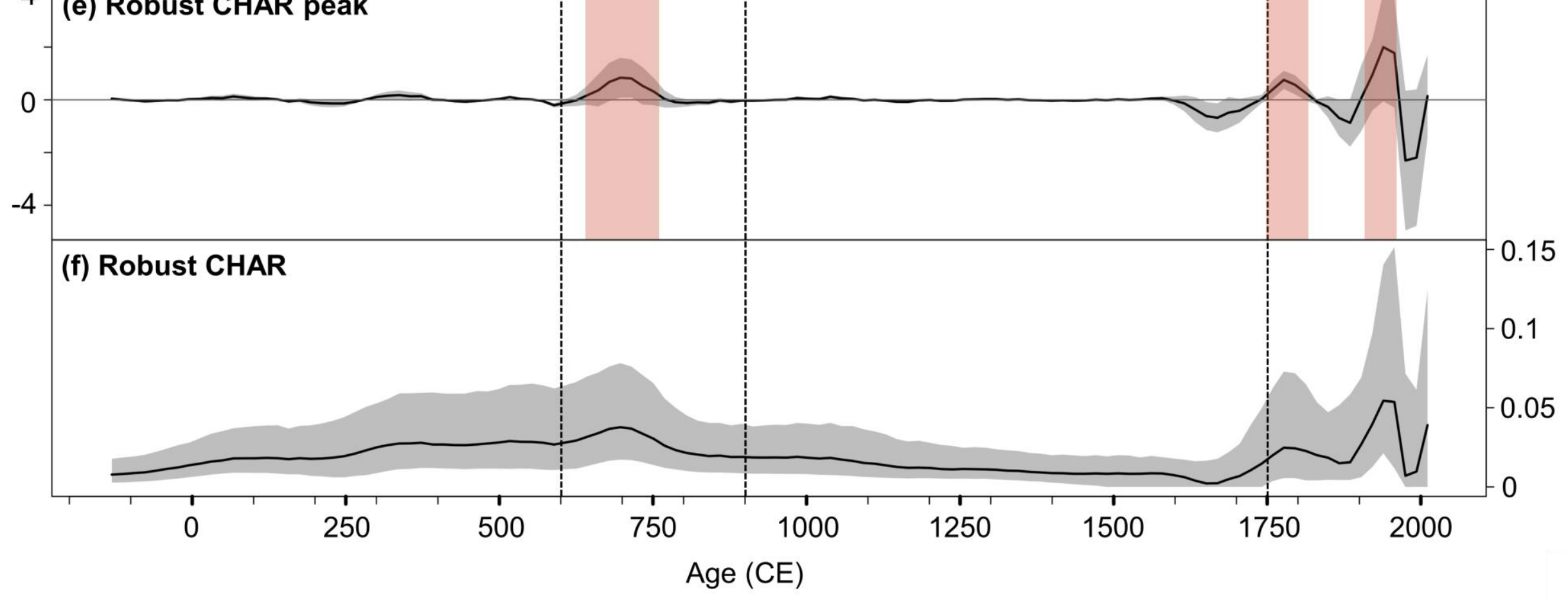

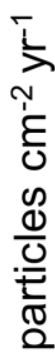

